\title{
Ultrasonography findings in immunoglobulin G4-related lymphadenopathy: a report of two cases
}

\author{
Tsuyoshi Morisaki ${ }^{1,2}$, Takahiro Fukuhara ${ }^{1}$, Eriko Matsuda ${ }^{1}$, Sueyoshi Moritani ${ }^{2}$, Naritomo \\ Miyake $^{1,2}$, Satoshi Koyama ${ }^{1}$, Kazunori Fujiwara ${ }^{1}$, Hiromi Takeuchi ${ }^{1}$
}

${ }^{1}$ Department of Otolaryngology-Head and Neck Surgery, Faculty of Medicine, Tottori University, Tottori, ${ }^{2}$ Center for Head and Neck Surgery, Kusatsu General Hospital, Shiga, Japan

\begin{abstract}
Lymphadenopathy is frequently observed in immunoglobulin G4 (IgG4)-related disease in some cases, and such cases are known as IgG4-related lymphadenopathy. The ultrasonography findings associated with IgG4-related lymphadenopathy have not been described in previous reports. Herein, we describe ultrasonography findings in two cases of IgG4-related lymphadenopathy in conjunction with histopathological findings. Combined ultrasound scanning with Doppler examination accurately represented the specific histopathological features of IgG4-related lymphadenopathy.
\end{abstract}

Keywords: immunoglobulin G4-related disease; lymphadenopathy; germinal center; ultrasonography

\section{Introduction}

Immunoglobulin G4-related disease (IgG4-RD) is a systemic condition characterized by the formation of masses in various organs that consist of lymphoplasmacytic infiltrates containing numerous IgG4-positive plasma cells. Lymphadenopathy, systemic or localized, is frequently observed (IgG4-related lymphadenopathy) $[1,2]$ and is divided into five subtypes based on the histopathological features [3].

Ultrasonographic findings of IgG4-related lymphadenopathy have not been published, especially due to the

Received 20.03.2019 Accepted 07.04.2019

Med Ultrason

2020, Vol. 22, No 1, 105-107

Corresponding author: Tsuyoshi Morisaki, MD, PhD

Department of Otolaryngology

Head and Neck Surgery, Faculty of Medicine,

Tottori University, 86, Nisi-cho, Yonago-shi,

Tottori, Japan, 683-0845

Phone: +81 859-38-6627

Email: tuyosi4755@gmail.com low frequency of this pathology. Therefore, reports describing ultrasonography findings in cases of IgG4-related lymphadenopathy are awaited with interest.

Herein, we describe the ultrasonography findings in two cases of IgG4-related lymphadenopathy (type III and type IV). Type IV is considered the most mon IgG4-related lymphadenopathy, followed by type III [4].

\section{Case reports}

\section{Case 1}

A 67-year-old woman presented with a right submandibular mass that had been growing over the previous 6 months. Blood analyses revealed a high level of serum soluble interleukin (IL)-2 receptor $(834 \mathrm{U} / \mathrm{ml})$. On the fluorine-18-fluorodeoxyglucose (FDG) positron emission tomography (PET) scan, an accumulation of FDG was seen in the swollen cervical and mediastinal lymph nodes.

Neck ultrasonography revealed swelling of multiple cervical lymph nodes and the maximum point-to-point 
lengths were $40 \mathrm{~mm}$ in the right submandibular region, $20 \mathrm{~mm}$ in the left submandibular region and $8-20 \mathrm{~mm}$ in both lateral neck regions. The lymph nodes were round in shape and internal echogenicity of the lymph nodes consisted of mostly low and diffusely mixed lace-like isoechogenicity. Fatty hila, which are usually observed as high echogenicity lines, were not observed (fig 1A). However, color Doppler examination detected slight blood flow signals in the center of the lymph nodes that seemed like fatty hila (fig 1B). The posterior echogenicity of the lymph nodes was mildly elevated. The interstitial tissue around the lymph nodes showed high echogenicity that indicated edematous condition.

Histological examination of the right submandibular lymph node revealed diffuse lymph follicle hyperplasia with each lymph follicle enlarged to two to five times the normal size, which indicated progressively transformed germinal centers (PTGCs) (fig 1C). The fatty hilum of the lymph node was invisible and numerous massive lymph follicles occupied the whole lymph node (fig 1D). Immunohistological examination of the lymph node sample revealed infiltration of IgG4-positive plasma cells. The ratio of $\mathrm{IgG} 4 / \mathrm{IgG}$ positive cells was $>40 \%$, with $>100$ IgG4-positive plasma cells per high-power light microscopy field. Subsequent blood analysis revealed high se- rum IgG4 (614 IU/ml). IgG4-related lymphadenopathy type IV/PTGC-type was diagnosed based on these findings, in accordance with previously described IgG4-RD diagnostic criteria [5].

\section{Case 2}

A 75-year-old woman was referred to our facility for a lymph node biopsy due to hypoalbuminemia $(2.9 \mathrm{~g} / \mathrm{dl})$ and hypergammaglobulinemia. Blood analyses revealed high serum soluble IL-2 receptor $(1726 \mathrm{U} / \mathrm{ml})$ and high serum IgG4 $(2190 \mathrm{mg} / \mathrm{dl})$. FDG PET scanning revealed accumulation of FDG in bilateral submandibular glands and swollen cervical, mediastinal, axillary, hilar, intraabdominal and inguinal lymph nodes.

Neck ultrasonography revealed a bilateral cervical lymph node swelling of 8-10 $\mathrm{mm}$ along the major axes. The lymph nodes were oval in shape and internal echogenicity was for the most part, uniformly low with obvious fatty hila in the centers of the lymph nodes (fig 2A). The surrounding interstitial tissue exhibited no abnormalities. High-resolution Doppler analysis using Advanced Dynamic Flow revealed diffusely arranged high blood flow spots in the cortexes of the lymph nodes (fig 2B).

Histopathological examination of the left cervical lymph node revealed that the basic structure of the lymph
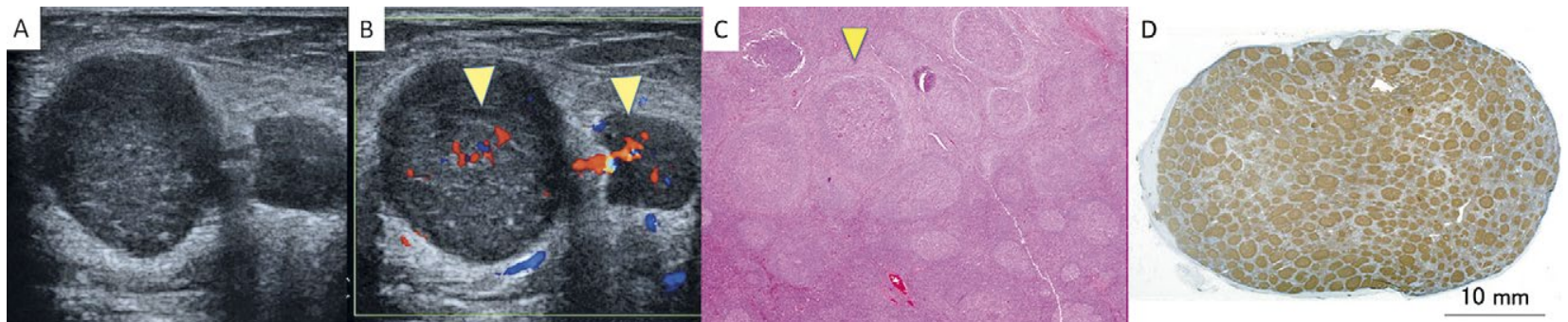

Fig 1. Neck ultrasonography and histopathological findings in case 1. B-mode ultrasound imaging revealed that the submandibular lymph nodes were round in shape and fatty hila were not present (A). On color Doppler examination, there were small amounts of blood flow signals in the center of the lymph nodes (B). Histopathological examination revealed lymph follicle hyperplasia with enlargement of each lymph follicle (arrows) (C; H\&E, $\times 20)$. Photograph of the lymph node showing numerous massive lymph follicles (D; CD-20 immunostaining, macrophotography).
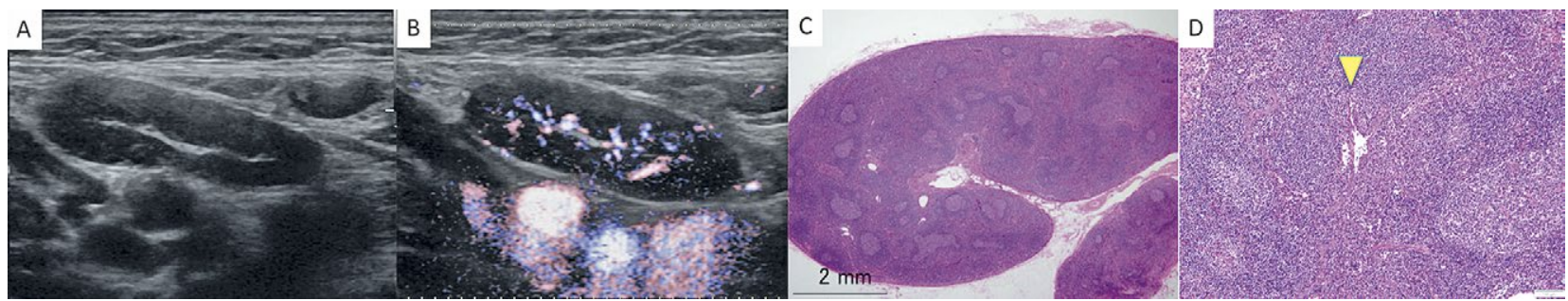

Fig 2. Neck ultrasonography and histopathological findings in case 2 . The lymph nodes were oval in shape, internal echogenicity was for the most part uniformly low, and fatty hila were observed in the centers of the lymph nodes (A). Doppler examination depicted diffusely arranged high blood flow spots in the cortexes of the lymph nodes (B). Histopathological examination showed that the basic structure of the lymph nodes largely remained, whereas gaps in the lymph follicles were expanded (C; H\&E, macrophotography) and contained noticeable blood vessels (arrow) (D; H\&E, ×40). 
node consisted of normal lymph follicles and the fatty hilum was mostly present (fig $2 \mathrm{C}$ ), whereas the gaps of the lymph follicles were expanded and contained noticeable blood vessels (fig 2D). Plasma cells infiltrated diffusely into the gaps of the lymph follicles. Immunohistological examination revealed that these plasma cells were mostly IgG4-positive. The ratio of IgG4/IgG-positive cells was $>90 \%$, and there were $>100$ IgG4-positive plasma cells per high-power light microscopy field. We diagnosed the patient with IgG4-related lymphadenopathy type III/interfollicular expansion and immunoblastosis according to the previously described criteria for IgG4-RD diagnosis [5].

\section{Discussions}

In case 1 , which was type IV IgG4-related lymphadenopathy, B-mode ultrasound imaging of the lymph node was suggestive of malignancy due to its round shape without a fatty hilum [6]. This finding corresponded to the histological finding of invisibility of the hilum. In contrast, Doppler analysis, which demonstrated a normal amount of blood flow in the center of the lymph node, indicated that the normal hilum was maintained without damage. Furthermore, diffuse or peripheral hypervascularity in the lymph nodes (which indicate malignancy) [7] were not observed on Doppler examination. In short, in type IV disease, B-mode ultrasound imaging was suggestive of malignancy but not Doppler analysis.

In case 2, which was type III IgG4-related lymphadenopathy, B-mode ultrasound imaging findings were similar to those of reactive lymphadenopathy with ovalshaped lymph nodes and obvious fatty hila in the center of the lymph nodes $[7,8]$. In contrast, Doppler analysis demonstrated high blood flow spots in the cortexes of the lymph nodes. These findings corresponded to the histological findings of a proliferation of blood vessels in the expanded gaps of the lymph follicles, which is not common in cases of reactive lymphadenopathy. In short, in type III disease, B-mode ultrasound imaging was suggestive of reactive lymphadenopathy but Doppler analysis was not.

In these two cases of IgG4-related lymphadenopathy, the combined use of morphological assessment via Bmode ultrasound imaging and blood flow assessment via
Doppler analysis enabled us to detect the internal structure of the lymph node accurately. Because the internal structure of the lymph node varies for each subtype of IgG4-related lymphadenopathy [3], ultrasonography findings should also be summarized individually for each subtype. Establishment of diagnostic ultrasonography findings for each subtype could make this diagnostic modality useful for the diagnosis of IgG4-lymphadenopathy.

In conclusion, in both the cases described above, ultrasonography findings accurately represented the specific histopathological features of each subtype of IgG4-related lymphadenopathy. Further reports describing ultrasonography findings in other subtypes of IgG4-related lymphadenopathy are awaited with interest.

\section{Acknowledgements}

This work was supported by JSPS KAKENHI Grant Number JP18K16889.

\section{References}

1. Sato Y, Kojima M, Takata K, et al. Systemic IgG4-related lymphadenopathy: a clinical and pathologic comparison to multicentric Castleman's disease. Mod Pathol 2009;22:589599.

2. Cheuk W, Yuen HK, Chu SY, Chiu EK, Lam LK, Chan JK. Lymphadenopathy of IgG4-related sclerosing disease. Am J Surg Pathol 2008;32:671-681.

3. Sato Y, Yoshino T. IgG4-related lymphadenopathy. Int J Rheumatol 2012;2012:572539.

4. Sato Y. IgG4-related disease in lymph nodes. Modern Physician 2015;35:1351-1353.

5. Okazaki K, Umehara H. Are classification criteria for IgG4RD now possible? The concept of IgG4-related disease and proposal of comprehensive diagnostic criteria in Japan. Int J Rheumatol 2012;2012:357071.

6. Ying M, Bhatia KS, Lee YP, Yuen HY, Ahuja AT. Review of ultrasonography of malignant neck nodes: greyscale, Doppler, contrast enhancement and elastography. Cancer Imaging 2014;13:658-669.

7. Ahuja A, Ying M. An overview of neck node sonography. Invest Radiol 2002;37:333-342.

8. Tschammler A, Wirkner H, Ott G, Hahn D. Vascular patterns in reactive and malignant lymphadenopathy. Eur Radiol 1996;6:473-480. 